\title{
Efecto de la densidad de siembra sobre el crecimiento de plantas de rábano (Raphanus sativus L.) bajo invernadero
}

\section{Effect of planting density on the growth of radish (Raphanus sativus L.) plants under greenhouse conditions}

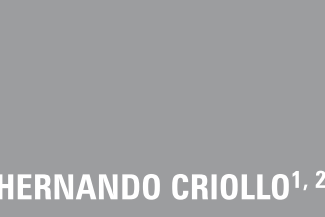 \\ JAVIER GARCÍA ${ }^{2}$}

\begin{tabular}{l} 
Raíces de rábano var. Crimson \\
Giant. \\
Foto: H. Criollo \\
\hline
\end{tabular}

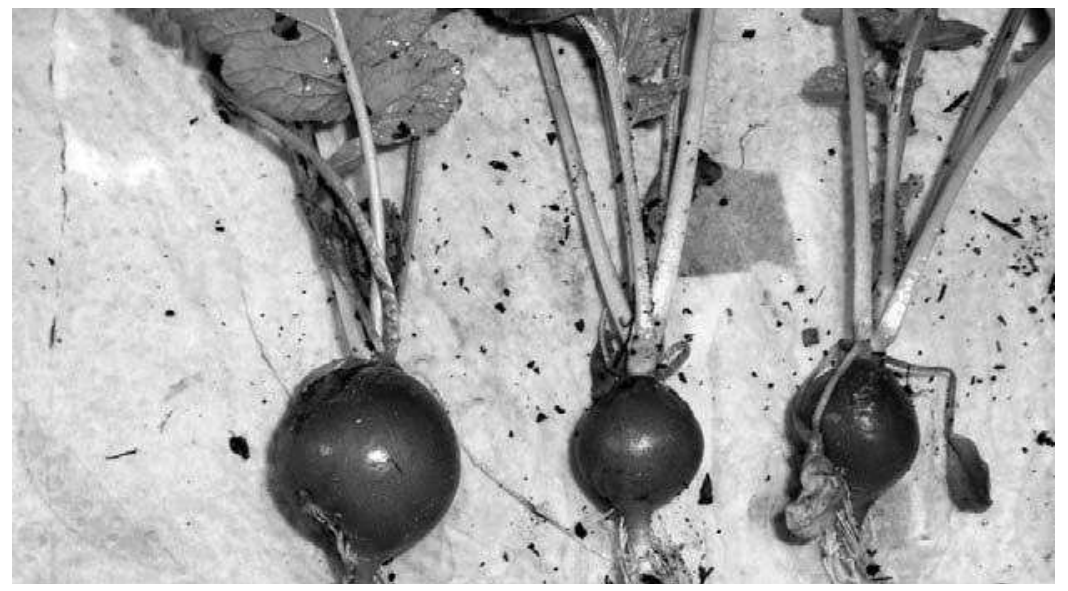

\section{RESUMEN}

El análisis de crecimiento de las plantas se ha desarrollado durante las últimas décadas como una disciplina, relacionada con la ecofisiología y la agronomía, con sus propios conceptos, términos y herramientas de cálculo. El presente trabajo se realizó en la Sabana de Bogotá y bajo condiciones de invernadero con el objeto de analizar el efecto de diferentes densidades de siembra (1, 2, 3 y 4 plantas por maceta de $201 \mathrm{~cm}^{2}$ ) sobre el crecimiento y producción de plantas de rábano. Mediante evaluaciones realizadas a partir de los 12 días y cada 4 días, se analizó la dinámica en el crecimiento del área foliar, diámetro de raíces, peso fresco de raíces, índice de área foliar, tasa de asimilación neta, tasa de crecimiento del cultivo, tasa relativa de crecimiento y el rendimiento. Las densidades de siembra afectaron la dinámica del crecimiento de las plantas de rábano, incrementándose hasta los 24 días después del trasplante, época en que se redujo su velocidad indicando el inicio de la madurez. Además, las bajas densidades incrementaron el área foliar y el rendimiento por planta pero redujeron la producción por área. La producción más alta $(28 \mathrm{~g} /$ maceta) se alcanzó con las mayores densidades de siembra (3 y 4 plantas/maceta).

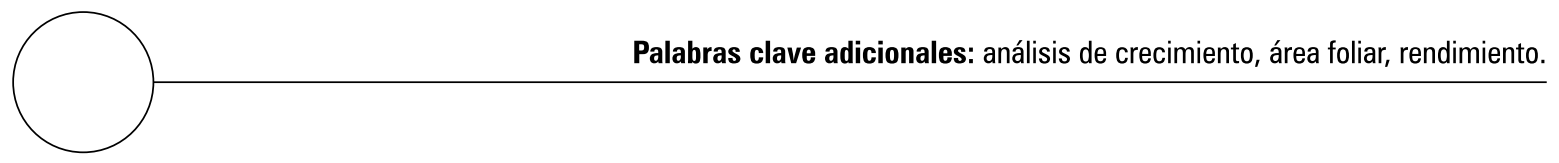

Facultad de Ciencias Agrícolas, Universidad de Nariño, Pasto (Colombia).

Autor para correspondencia. hcriolloescobar@gmail.com 


\section{ABSTRACT}

The plant growth analysis has been developed over the decades as a discipline related to the ecophysiology and agronomy, with their own concepts, terms and calculation tools. In the Bogota Plateau and under greenhouse conditions this study was carried out in order to analyze the effect of different densities (1, 2, 3 or 4 plants per $201 \mathrm{~cm}^{2}$ pot) of sowing on growth and yield of plants of radish. The measurements were carried out starting from 12 days alter planting and every four days in order to analyze the dynamics in growth of leaf area, diameter of roots, fresh weight of roots, leaf area index, net assimilation rate, crop growth rate, relative growth rate, and yield/area. The planting densities affected rate dynamics of growth of radish plants, increasing to 24 days after transplanting, at which time its speed was reduced, indicating the onset of maturity. In addition, low densities increased the leaf area and yield per plant but reduced the yield/area. The highest production ( $28 \mathrm{~g} / \mathrm{pot}$ ) was achieved with the highest densities ( 3 and 4 plants/pot).

Additional key words: growth analysis, leaf area, yield.

Fecha de recepción: 18-09-2009

Aprobado para publicación: 30-11-2009

INTRODUCCIÓN

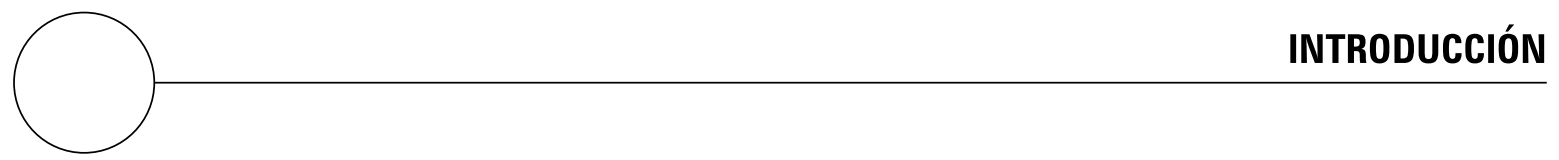

El rábano (Raphanus sativus L.) es una planta de gran importancia por sus propiedades farmacéuticas y altos contenidos vitamínicos y de minerales; $100 \mathrm{~g}$ de materia fresca de rábano contienen 0,86 $\mathrm{g}$ de prótidos, $30 \mathrm{UI}$ (unidades internacionales) de vitamina $\mathrm{A}, 30 \mathrm{mg}$ de vitamina $\mathrm{B} 1,20 \mathrm{~g}$ de vitamina B2 y 24 mg de vitamina C. Presenta además un contenido de $37 \mathrm{mg}$ de $\mathrm{Ca}, 31 \mathrm{mg}$ de $\mathrm{P}$ y $1 \mathrm{mg}$ de Fe (Ramírez y Pérez, 2006).

Esta planta Brassicaceae se supone originaria del Japón o China. Es una planta anual, de raíz gruesa y carnosa, de tamaño y forma variable, piel de color rojo, rosado, blanco u oscuro, según la variedad; posee hojas basales, pecioladas, lámina lobulada con uno a tres pares de segmentos laterales con bordes dentados (Casimir, 2001).

El rábano se desarrolla bien en climas medios, aunque las altas temperaturas pueden originar sabores picantes en sus raíces. Su ciclo productivo es corto y puede variar entre 20 y $70 \mathrm{~d}$, según la variedad, con una temperatura óptima de 18 a $22^{\circ} \mathrm{C}$; se adapta a cualquier tipo de suelo pero los suelos profundos, arcillosos y de reacción neutra son los ideales (Montero et al., 2006).

Según Rincón et al. (2007), el crecimiento y la capacidad productiva de un cultivo es el resultado del genotipo, del ambiente que lo rodea y de su interacción. El genotipo es relativamente constante si se compara con la variabilidad del ambiente; sin embargo, la expresión fenotípica es ampliamente influenciada por los cambios ambientales y cualquier variable que produzca efectos sobre el medio va a verse reflejada en el crecimiento y productividad del cultivo (Marín, 1986).

El análisis de crecimiento es ahora una herramienta ampliamente usada en áreas tan diferentes como en el fitomejoramiento, la fisiología de los cultivos y en la ecología de las plantas (Poorter y Garnier, 1996). Se considera que el análisis de crecimiento representa el primer paso en el análisis de la productividad primaria, siendo un enlace entre el registro de la producción vegetal 
y su investigación por métodos fisiológicos, pudiendo ubicarse consecuentemente dentro del ámbito de los estudios ecofisiológicos. Su ventaja radica en la facilidad de obtención de los datos en los cuales se basa, como son el peso seco de plantas completas o de sus partes (hojas, tallos, vástagos) y las dimensiones del aparato asimilatorio (área foliar, área de hojas y tallos, contenido de clorofila, etc.) (Marín, 1989; Kvet et al., 1971).

El rábano es un cultivo de manejo intensivo del cual hay muy poca información local sobre análisis de crecimiento y fenología; los análisis detallados del crecimiento de las plantas permiten cuantificar aspectos como la duración del ciclo, definición de estados fenológicos y estados de desarrollo, distribución de asimilados en los diferentes órganos (Azofeifa y Moreira, 2004). Además, los análisis de crecimiento son esenciales para lograr una mejor comprensión de los procesos fisiológicos que definen la producción vegetal y así definir las mejores alternativas de manejo del cultivo en aspectos relacionados con la fertilización, riego, prácticas sanitarias, podas, orientación del cultivo y densidad de siembra, entre otros (Lambers y Poorter, 1992; Barrientos, 1988).

Taiz y Zeiger (2006) definen el crecimiento como un incremento constante en el tamaño de un organismo determinado por procesos de morfogénesis y diferenciación; el primero es el desarrollo de la forma o modelo de la célula u órgano, mientras que el segundo es el proceso por el cual las células cambian estructural y bioquímicamente para formar o adquirir funciones especializadas. Ambos procesos se pueden medir mediante la tasa absoluta de crecimiento en función de la cantidad de materia seca y la tasa de funcionamiento de esta, en relación con la influencia del ambiente (Milthorpe y Moorby, 1982). Lambert et al. (1998) definen el crecimiento como un incremento en masa seca, volumen, longitud o área y, en alto grado, involucra la división, expansión y diferenciación celular. Öpik y Rolfe (2005) describen que el proceso de crecimiento, a un nivel del organismo, significa la multiplicación coordinada, incremento de tamaño y especialización de millones de células, todas ordenadas en posiciones exactas.

Es importante poder expresar la producción de un cultivo en términos de crecimiento. El análisis de crecimiento trata de explicar en términos matemáticos las variaciones de peso seco y del área foliar en función del tiempo. La estimación de los índices de eficiencia en el crecimiento requiere de la medición del peso seco total de las plantas, así como de sus diferentes órganos y área foliar, en intervalos de tiempo durante el desarrollo de la planta (Radford, 1967; Hunt, 1990); estas mediciones brindan una información más precisa acerca de la eficiencia de las plantas en la acumulación $y$ transporte de asimilados que las mediciones de índole agronómica (Borrego et al., 2000).

Mediante el uso de prácticas agrícolas se provee a los cultivos de las condiciones más favorables para la expresión del mayor rendimiento potencial; dentro de dichas prácticas se destaca el manejo de la densidad de población. A través de esta práctica agrícola pueden incrementarse la producción de biomasa y el rendimiento de los cultivos, debido al aumento en el área foliar, el índice de área foliar y la duración de la misma, ocasionado por el mayor número de hojas por unidad de superficie (Olalde et al., 2000; Viloria, 1998 y Cebula, 1995), ya que el tamaño y la duración del aparato fotosintético están relacionados con el rendimiento. Asimismo, el mayor crecimiento del dosel vegetal proporciona una mayor intercepción de luz, lo cual incrementa la fotosíntesis y producción de biomasa como resultado de un mayor aprovechamiento de los recursos hídricos y nutrimentales (Aguilar et al., 2005).

Con base en estas consideraciones, el presente trabajo se planteó con el objetivo de evaluar el efecto de cuatro densidades de siembra sobre el comportamiento del rábano, mediante el análisis de crecimiento. 


\section{MATERIALES Y METODOS}

\section{Localidad y material vegetal}

Este trabajo se realizó en un invernadero de plástico de la Facultad de Agronomía de la Universidad Nacional de Colombia, Bogotá, con una temperatura y humedad relativa promedias en el interior de $15,6^{\circ} \mathrm{C}$ y $61 \%$, respectivamente.

Se utilizaron semillas de rábano variedad Crimson Giant que se germinaron en bandejas plásticas de 72 alvéolos con turba canadiense. Una vez aparecieron las primeras hojas verdaderas (15 días después de la siembra), las plántulas se transplantaron a macetas plásticas de polipropileno de color rojo, de 2 L de capacidad y de 201 $\mathrm{cm}^{2}$ de área superficial; el sustrato utilizado fue suelo de textura arenosa.

Los tratamientos correspondieron a cuatro densidades de siembra: a) 1 planta/maceta (497.512 plantas/ha); (b), 2 plantas/maceta (995.024 plantas/ha); (c) 3 plantas/maceta (1.492.536 plantas/ ha), y (d) 4 plantas/maceta (1.990.048 plantas/ ha). Las macetas se organizaron en el terreno de acuerdo a un diseño de bloques completos al azar (BCA) con cuatro repeticiones, teniendo en cuenta su ubicación en el invernadero; cada repetición estuvo conformada por 10 macetas. Antes de la siembra, se le aplicó al suelo fertilizante de grado 0-10-15 suplementado con elementos menores y secundarios $\left(1.000 \mathrm{~kg} \mathrm{ha}^{-1}\right)$ más urea en dosis de $100 \mathrm{~kg} \mathrm{ha}^{-1}$.

\section{Evaluación del crecimiento y rendimiento}

A partir del establecimiento de las plantas en las macetas (12 días después del transplante [ddt]) y cada $4 \mathrm{~d}$, se extrajeron de cada bloque muestras de plantas correspondientes a cada tratamiento, para determinar en cada evaluación el número de hojas $(\mathrm{NH})$, área foliar (AF), diámetro de raíz (DR), peso fresco de raíz (PFR), peso seco de hojas (PSH), peso seco de raíz (PSR) y peso seco total (PST). El área foliar se determinó con un medidor LICOR-3000, el diámetro con un calibrador de vernier, el peso fresco con una balanza analítica y el peso seco después del secado de muestras en un horno a $70^{\circ} \mathrm{C}$ durante $72 \mathrm{~h}$.

Con los datos anteriores de área foliar y peso seco se procedió al cálculo los siguientes índices de crecimiento:

Índice de área foliar (IAF): corresponde al área foliar de la planta sobre el área foliar del suelo que ocupa (Hunt, 1990).

$$
I A F=\frac{A F_{\text {planta }}}{A_{\text {suelo }}}
$$

Tasa absoluta de crecimiento (TAC): corresponde al incremento de peso seco de la planta o de cada uno de sus órganos por unidad de tiempo (Hunt, 1990).

$$
T A C=\frac{W_{2}-W_{1}}{T_{2}-T_{1}}
$$

Tasa relativa de crecimiento (TRC): permite medir la eficiencia de la planta en el incremento de peso por unidad de tiempo (Hunt, 1990).

$$
T R C=\frac{\operatorname{Ln} W_{2}-\operatorname{Ln} W_{1}}{T_{2}-T_{1}}
$$

Tasa de asimilación neta (TAN): mide la eficiencia fotosintética y determina el incremento de peso por unidad de área foliar en una unidad de tiempo y se calculó mediante la fórmula propuesta por Gómez et al. (1999).

$$
T A N=\frac{W_{2}-W_{1}}{T_{2}-T_{1}} \times \frac{L n A F_{2}-L n A F_{1}}{A F_{2}-A F_{1}}
$$

Tasa de crecimiento del cultivo (TCC): mide los incrementos de biomasa seca por unidad de área de suelo en una unidad de tiempo (Radford, 1967).

$$
T C C=I A F \times T A N
$$

El rendimiento se calculó como el peso de raíces obtenidas en cada maceta ( $\mathrm{g} /$ maceta). 


\section{Análisis estadístico}

El diámetro de raíces y el PFR, evaluadas al final del trabajo, se sometieron a análisis de varianza y pruebas de comparación de medias de Tukey $(P \leq 0,05)$, utilizando el paquete estadístico SAS. El comportamiento del crecimiento del rábano se evaluó mediante análisis de regresión tomando el tiempo como variable independiente; los modelos se seleccionaron con base en los mejores valores de $R^{2}$ y basados en el reporte del paquete estadístico CurveExpert versión 1.3.

\section{RESULTADOS Y DISCUSIÓN}

\section{Área foliar por planta (AF)}

El comportamiento del AF mostró una tendencia ascendente en las primeras evaluaciones, tendencia que decreció al final del estudio en los tratamientos evaluados con excepción del tratamiento correspondiente a 1 planta/maceta, en donde el incremento en el número de hojas con- tinuó. Igualmente, los tratamientos con menor densidad permitieron una mayor AF (figura 1). Según Clavijo (1989), cuando se siembran diferentes densidades de plantas, su respuesta a los factores ambientales difiere según la densidad de población.

También, estas observaciones coinciden con Jolliffe y Gaye (1995) quienes afirman que un rápido crecimiento y una mayor expansión de hojas y raíces se presenta cuando no hay otras plantas competidoras en la cercanía; cuando hay mayor densidad, una planta que crece más rápido que su vecina utilizará una mayor cantidad de un determinado recurso disponible e incrementará su tasa de crecimiento en general. La mayor extensión de las hojas permitirá a la planta poseer una mayor área de interceptación de luz y una mayor producción fotosintética por planta.

\section{Diámetro de la raíz}

La tasa de crecimiento de la raíz en tamaño es un aspecto importante si se considera que la co-

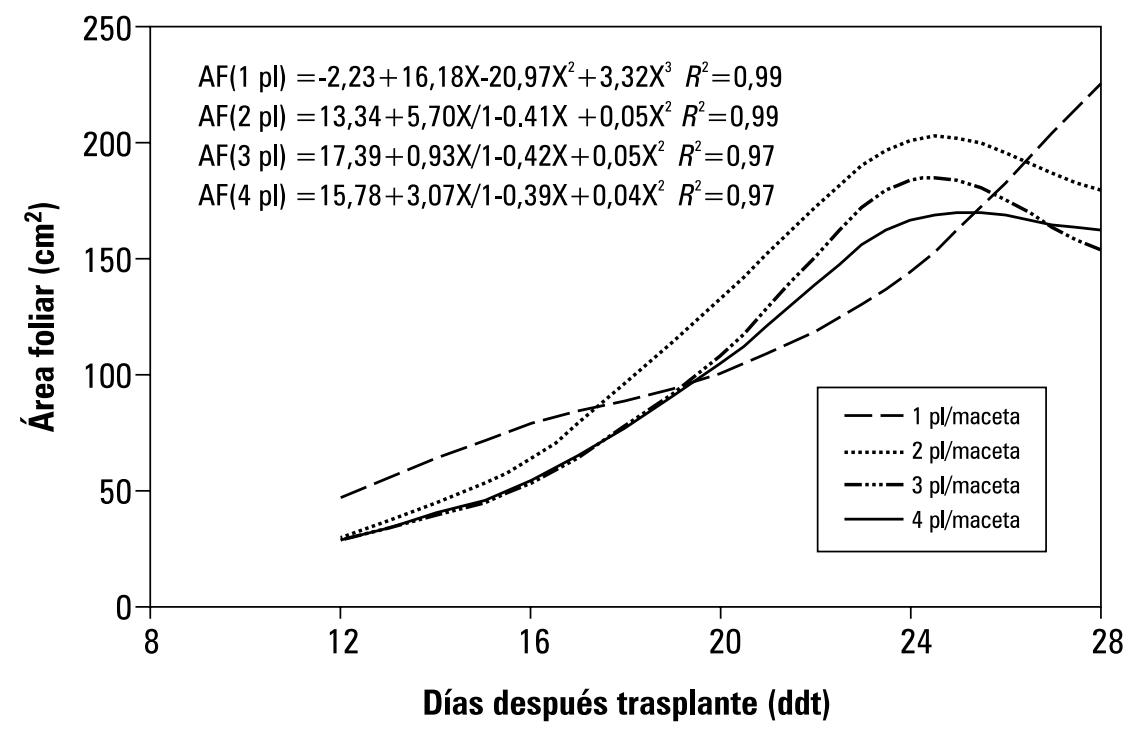

Figura 1. Comportamiento del área foliar en plantas de rábano a través del tiempo y en cuatro densidades de siembra. 
mercialización de este producto es en fresco. En el tratamiento de 1 planta/maceta se observó un incremento sostenido durante todo el experimento (figura 2), lo cual indica un mayor tamaño final del producto y presenta correlación con el comportamiento del AF. Los tratamientos con 2, 3 y 4 plantas por maceta mostraron una curva de crecimiento de tipo sigmoideo, con un crecimiento inicial lento, una segunda fase de crecimiento acelerado motivado por la rápida asignación y traslocación de fotoasimilados hacia la raíz y un descenso al iniciarse el periodo de maduración causado posiblemente por los bajos gradientes entre los fotoasimilados de la raíz y de las hojas (Cruz-Huerta et al., 2005).

La evaluación del diámetro final de las raíces de rábano mostró que la siembra de 1 planta/maceta permitió un mayor diámetro de raíces $(3,03$ $\mathrm{cm})$, con diferencias significativas en comparación con los demás tratamientos (tabla 1). En general, un menor número de plantas/área significó mayor crecimiento radical.

El menor diámetro observado en tratamientos con mayor densidad puede explicarse en la ma- yor competencia intraespecífica que se da por nutrientes, espacio y radiación, generada por el más alto número de plantas por maceta, lo cual genera una menor cantidad de asimilados por planta para ser particionados hacia las raíces (Páez et al., 2000).

\section{Peso fresco de raíces}

El peso fresco de raíces de rábano estuvo determinado por la densidad de siembra. Los mayores valores al final de las evaluaciones se alcanzaron en el tratamiento 1 planta/maceta con $15 \mathrm{~g} /$ raíz comparados con las 2, 3 y 4 plantas por maceta,

\section{Tabla 1. Diámetro y rendimiento de raíces de rábano, obtenidos con diferentes densidades de siembra.}

\begin{tabular}{|c|c|c|}
\hline $\begin{array}{c}\text { Plantas por } \\
\text { maceta }\end{array}$ & Diámetro $(\mathrm{cm})$ & $\begin{array}{c}\text { Rendimiento } \\
\text { (g/maceta) }\end{array}$ \\
\hline 1 & $3,03 \mathrm{a}$ & $28,0 \mathrm{a}$ \\
\hline 2 & $2,53 \mathrm{~b}$ & $27,9 \mathrm{a}$ \\
\hline 3 & $2,40 \mathrm{bc}$ & $21,5 \mathrm{~b}$ \\
\hline 4 & $2,13 \mathrm{c}$ & $15,0 \mathrm{c}$ \\
\hline
\end{tabular}

Promedios con letras distintas indican diferencia significativa según la prueba de Tukey $(P \leq 0,05)$.

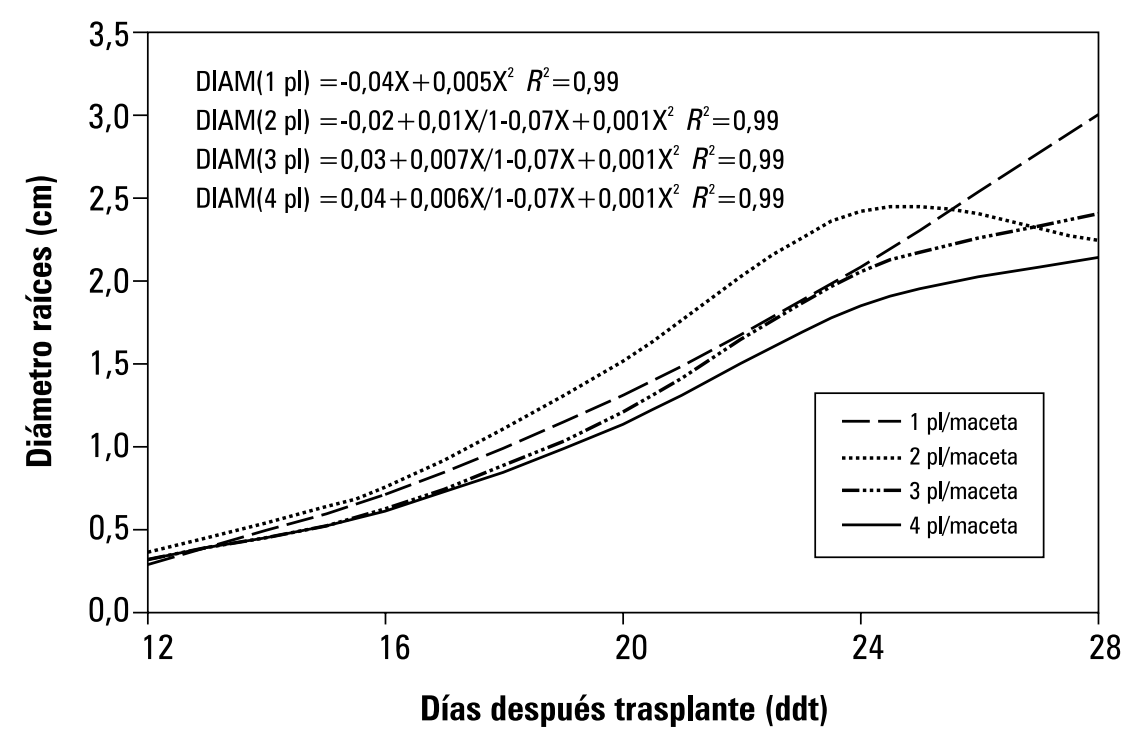

Figura 2. Diámetro de raíces en plantas de rábano a través del tiempo y en cuatro densidades de siembra. 
con pesos de 10,73 g, 9,33 g y 7,97 g, respectivamente (figura 3). Según Gardner et al. (1985), al incrementarse la densidad de población de un cultivo, generalmente disminuye la biomasa por planta pero se incrementa por unidad de superficie, lo cual concuerda con el presente estudio. Así mismo, estos resultados coinciden con Pérez et al. (2005) quienes manifiestan que las altas densidades de plantación reducen el crecimiento de las variables vegetativas como área foliar, número de hojas y materia seca, siendo más notorios estos efectos al final del cultivo.

\section{Índice de área foliar (IAF)}

Una de las funciones más importantes de la hoja es la absorción lumínica, por lo cual esta función se explica mejor con el área foliar desarrollado (Bresinsky et al., 2008). Las mayores densidades de siembra dieron origen a mayores IAF, indicando una mayor área foliar por unidad de área de suelo y con excepción de 1 planta/maceta, los mayores IAF se presentaron en la cuarta evaluación. El tratamiento 1 planta/maceta presentó los menores IAF $(1,1)$, aunque no se logró alcanzar los máximos durante el periodo de evaluación (figura 4). El mayor IAF se alcanzó sembrando 4 plantas/maceta con un valor de 3,4, el cual se considera ideal para una planta tipo C3; en su orden le siguieron los tratamientos con 3 y 2 plantas/maceta con un IAF de 2,8 y 2,0 , respectivamente.

Estos resultados coinciden con Gardner et al. (1985), Cebula (1995) y Jolliffe y Gaye (1995), quienes indican que aunque el incremento en la densidad de población ocasiona una disminución en tamaño, vigor y peso de la planta, la biomasa por unidad de superficie se incrementa hasta un máximo, que para la mayoría de plantas de cultivo con mecanismo fotosintético C3, se alcanza con un IAF entre 3 y 4 , valores alcanzados con 4 plantas/maceta en este estudio. Igualmente, Kerby et al. (1990) encontraron que en algodón, las mayores densidades de siembra produjeron los máximos valores de IAF, en momentos en que el cultivo debe destinar una creciente cantidad de fotoasimilados al llenado de frutos, por lo que se necesita interceptar la mayor cantidad de radiación posible.

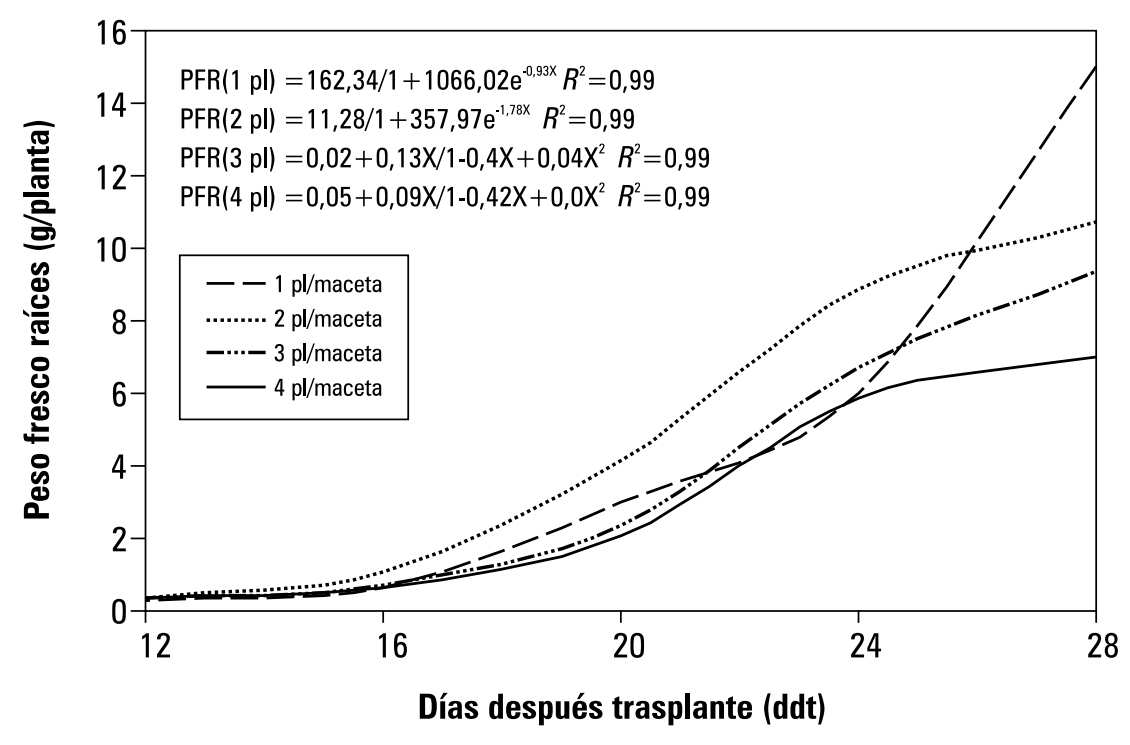

Figura 3. Peso fresco de raíces de rábano a través del tiempo y en cuatro densidades de siembra. 


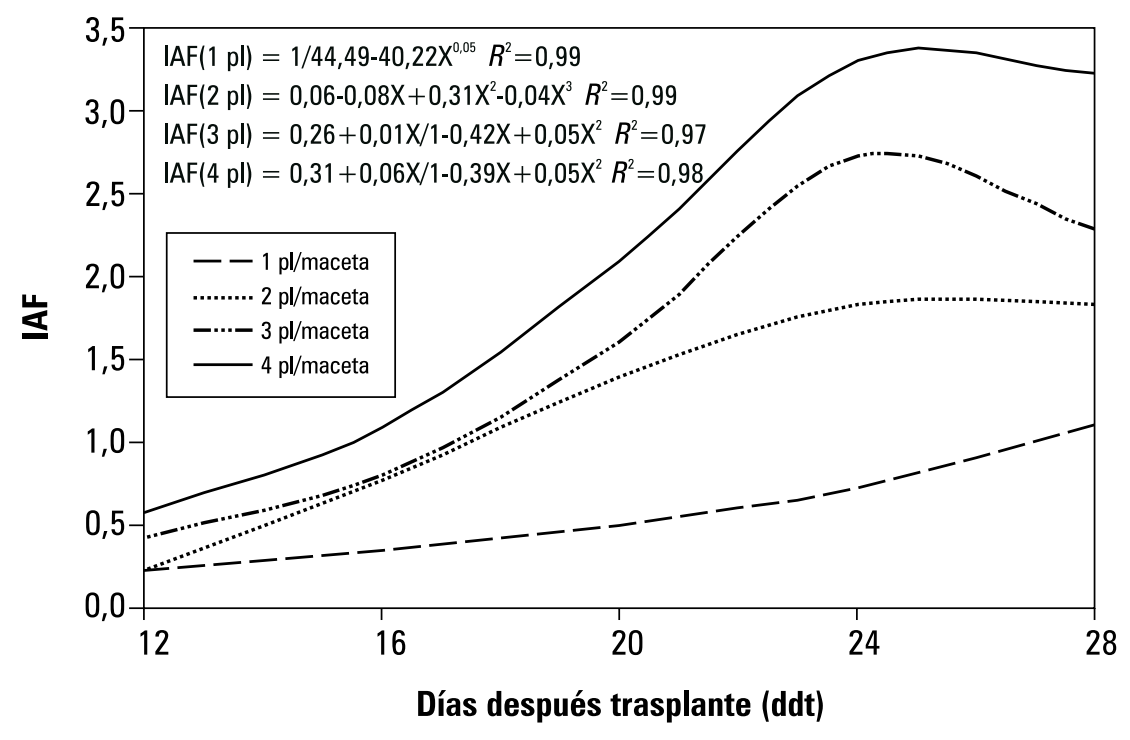

Figura 4. Comportamiento del índice de área foliar (IAF) en plantas de rábano a través del tiempo y en cuatro densidades de siembra.

\section{Tasa de asimilación neta (TAN)}

Los valores de TAN mostraron amplia variación en cada una de las densidades estudiadas, a través del tiempo de evaluación. Mientras que los mayores valores para los tratamientos de $3(2,01$ $\left.\mathrm{g} \mathrm{dm}^{-2} \mathrm{~d}^{-1}\right)$ y 4 plantas/maceta $\left(1,57 \mathrm{~g} \mathrm{dm}^{-2} \mathrm{~d}^{-1}\right)$ se presentaron en la cuarta lectura (16 ddt), el tratamiento con 2 plantas/maceta alcanzó su valor máximo a los 12 ddt (tercera lectura) y el tratamiento con 1 planta/maceta en la última lectura con $2,81 \mathrm{~g} \mathrm{dm}^{-2} \mathrm{~d}^{-1}$ (figura 5).

Estas variaciones pueden presentarse por la diferencia en los periodos de competencia entre plantas, en su autosombreamiento y principalmente por las diferencias en el inicio del engrosamiento acelerado de la raíz, que da origen a fuertes gradientes en los potenciales fuente-vertedero ya que según Pollock y Farrar (1996) y Shibles (1987), los máximos valores de TAN se alcanzan por las altas demandas de fotoasimilados que se presentan cuando un órgano como el fruto o las raíces, en el caso del rábano, inicia el proceso de llenado; igualmente, cuando el órgano alcanza su madurez, los valores de la TAN se reducen drás- ticamente. Además existen otros factores determinantes de estas variaciones, como los mencionados por Milthorpe y Moorby (1982) y Shibles (1987) y relacionados con la ontogenia del cultivo. Estos autores mencionan entre otros factores, el sombreo de las hojas superiores sobre las inferiores, la reducción de la capacidad fotosintética de las últimas hojas formadas debido a su baja concentración clorofílica y niveles de proteínas solubles, el menor estímulo de los vertederos.

Así mismo, Poorter (1989) manifiesta que el componente fisiológico del crecimiento como es la TAN, es el resultado del balance neto entre las ganancias por la tasa de fotosíntesis y las pérdidas por las tasas de respiración de hojas, tallos y raíces y que en su definición también intervienen otros factores como la distribución de biomasa en los diferentes órganos, la composición química y la formación de área foliar (Villar et al., 2004).

\section{Tasa de crecimiento del cultivo (TCC)}

Los valores más altos pueden observarse en la cuarta lectura para los tratamientos con altas 


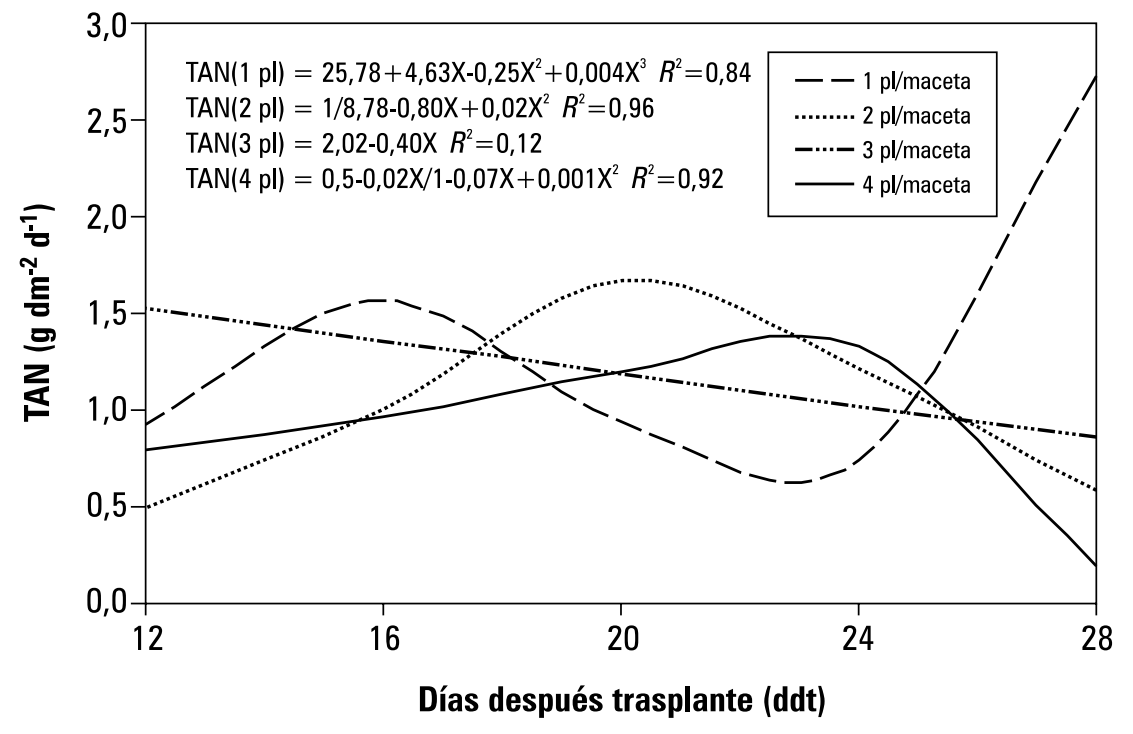

Figura 5. Comportamiento de la tasa de asimilación neta (TAN) en plantas de rábano a través del tiempo y en cuatro densidades de siembra.

densidades poblacionales $\left(5,59 \mathrm{~g} \mathrm{dm}^{-2} \mathrm{día}^{-1}\right) \mathrm{y}$ $5,32 \mathrm{~g} \mathrm{dm}^{-2}$ día $^{-1}$ para los tratamientos de 3 y 4 plantas/maceta, respectivamente; el tratamiento con $1 \mathrm{planta} /$ maceta alcanzó su mayor valor en la última evaluación debido a la menor interferencia con otras plantas, lo cual le permitió un mayor crecimiento foliar (figura 6).

La disminución final en la TCC puede atribuirse, de acuerdo con Borrego et al. (2000), al inicio de la senescencia foliar y al menor potencial de vertedero presentado en la etapa de madurez de las raíces del rábano; igualmente se puede afirmar que la densidad de siembra cumple un papel importante en la definición de la TCC ya que afecta directamente el comportamiento del IAF y de la eficiencia fotosintética, medida como TAN.

\section{Tasa relativa de crecimiento (TRC)}

Este parámetro indica la acumulación de biomasa presente por unidad de biomasa producida (Borrego et al., 2000). El comportamiento observado de esta variable durante el periodo experimental, permite observar variaciones determinadas por el comportamiento de la TAN (figura 7). Du- rante sus primeros estadios, el crecimiento suele tener una dinámica exponencial y suele reflejar diferencias significativas entre especies o manejo agronómico diferente.

A diferencia de la TAN, en la primera lectura se observa en todos los tratamientos una gran eficiencia en la conversión de peso seco por unidad de peso, debido posiblemente a la mayor relación que se presenta entre el AF y el peso de las hojas en las primeras fases del crecimiento y a que los tejidos jóvenes poseen mayor actividad biológica y capacidad de síntesis. Además, las tendencias representadas en la figura 7 concuerdan con lo planteado por Blackman (1986) y encontrado por varios autores en torno a los cambios temporales en el TCR, es decir, los índices son mayores durante las fases vegetativas tempranas de las plantas y disminuyen conforme se alcanza la madurez y las hojas llegan a la senescencia.

\section{Rendimiento}

Los datos de rendimiento se expresan en peso de raíces por unidad de área $\left(201,06 \mathrm{~cm}^{2}\right)$; el rendimiento osciló entre 27,99 g/maceta en el tra- 


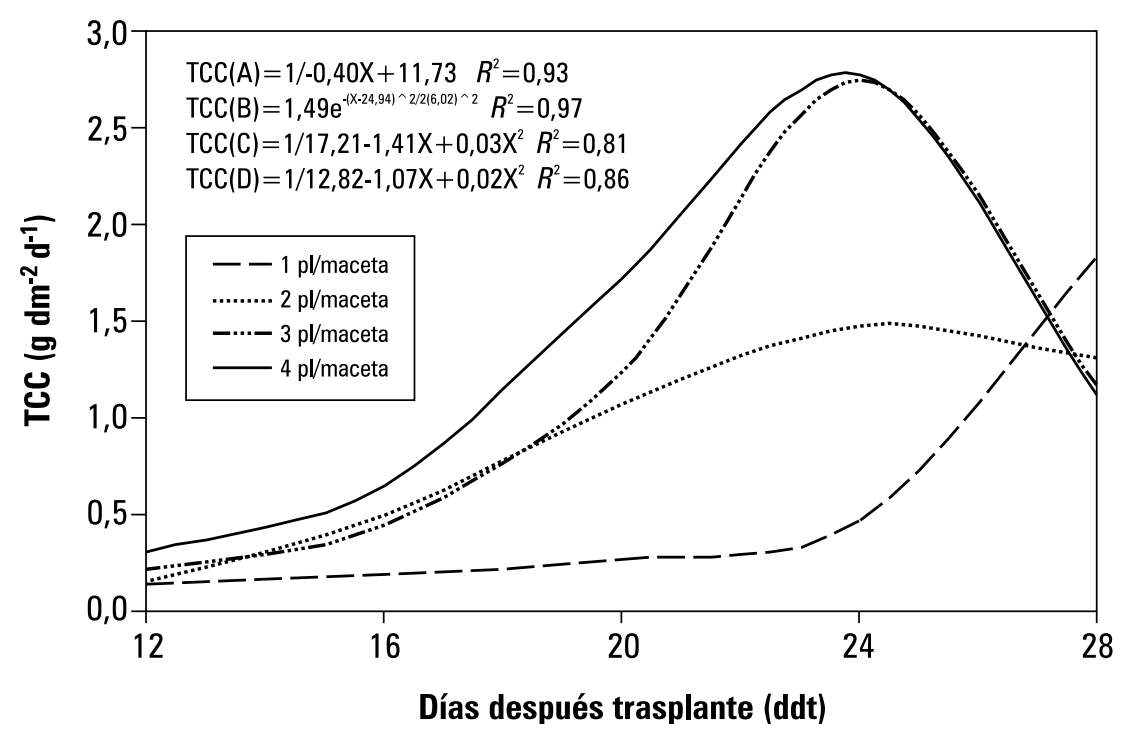

Figura 6. Comportamiento de la tasa de crecimiento del cultivo (TCC) en plantas de rábano a través del tiempo y en cuatro densidades de siembra.

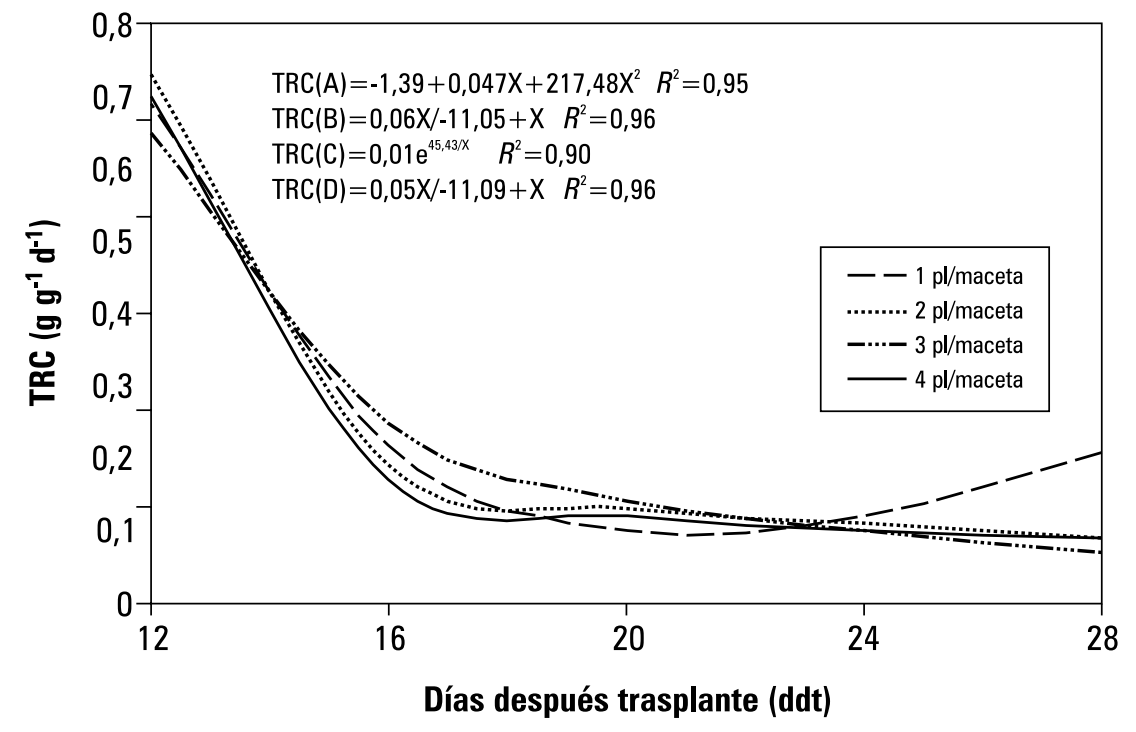

Figura 7. Comportamiento de la tasa relativa de crecimiento (TRC) en plantas de rábano a través del tiempo y en cuatro densidades de siembra. 
tamiento con 3 plantas y $14,99 \mathrm{~g} /$ maceta en el tratamiento con una planta por matera. Se determinaron diferencias estadísticas entre el rendimiento de los tratamientos con 3 plantas $(28,00$ g) y 4 plantas/maceta $(27,91 \mathrm{~g} /$ maceta $)$ con los demás tratamientos. Dos plantas por maceta $(21,48$ g) produjeron un rendimiento significativamente más alto que una planta por maceta $(15,0 \mathrm{~g})$, que fue el tratamiento menos productivo (tabla 1 ).

Estos resultados coinciden con lo expuesto por Gardner et al. (1985) quienes afirman que con el aumento de la densidad de población, por lo general, disminuye la biomasa por planta pero se incrementa por unidad de superficie. La relación entre las variables IAF, AF, PFR y rendimiento expresado como peso de raíces por maceta, cuando se trabaja con diferentes densidades de siembra, permite establecer que aumentando el IAF con densidad de plantas, se incrementa el rendimiento/maceta pero se reduce el AF y PFR por planta (figura 8).

\section{CONCLUSIONES}

- Las densidades de siembra afectaron el comportamiento del crecimiento del rábano.

- El IAF y la TCC se incrementaron con la densidad de población, alcanzándose los valores máximos en la lectura a los $24 \mathrm{ddt}$, época en que comienzan a estabilizarse las demás variables de crecimiento.

- Las bajas densidades de población se asociaron con mayor AF y mayor producción/planta pero con menores rendimientos/área.

- Una densidad de 4 plantas/maceta (equivalentes a 1.990 .049 plantas/ha) produjo los mayores rendimientos de raíces $(28,0 \mathrm{~g} / \mathrm{ma}-$ ceta), en comparación con 1 planta/maceta (497.512 plantas/ha) con un rendimiento de $15,0 \mathrm{~g} / \mathrm{maceta})$.

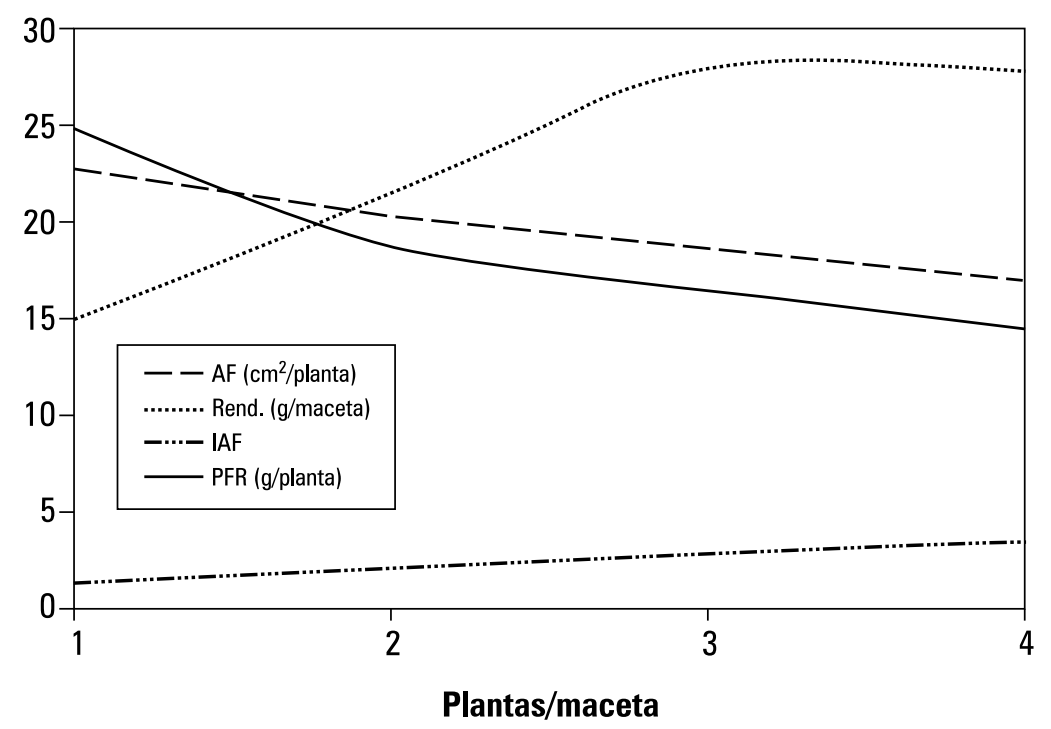

Figura 8. Relación entre las variables área foliar (AF), índice de área foliar (IAF), peso fresco por planta (PFR) y rendimiento por maceta en plantas de rábano en cuatro densidades de siembra. 


\section{REFERENCIAS BIBLIOGRÁFICAS}

Aguilar, L.; J. Escalante; L. Fucikovsky; L. Tijerina y E. Mark. 2005. Área foliar, tasa de asimilación neta, rendimiento y densidad de población en girasol. Terra Latinoamericana 23(3), 303-310.

Azofeifa, A. y M. Moreira. 2004. Análisis de crecimiento del chile jalapeño (Capsicum annuum L. cv. hot), en Alajuela, Costa Rica. Agron. Costarr. 28(1), 57-67.

Barrientos, E. 1988. Evaluación de necesidades de N, P y $\mathrm{Mg}$ en chile dulce, Capsicum annuum L., asociado con café, Coffea arabiga, en siembra nueva. Trabajo de grado. Escuela de Fitotecnia, Facultad de Agronomía, Universidad de Costa Rica, Centro Regional de Occidente, Costa Rica.

Blackman, G. 1986. The application of the concepts of growth análisis to the assessment of productivity. pp. 243-259. En: Eckard, F. (ed.). Functioning of terrestrial ecosystems of the primary production level. Proc. Copenhagen Symposium, Unesco, Copenhagen.

Borrego, F.; J. Fernandez; A. Lopez; V. Parga; M. Murillo y A. Carvajal. 2000. Análisis de crecimiento en siete variedades de papa (Solanum tuberosum L.). Agronomía Mesoamericana 11(1), 145-149.

Bresinsky, A.; C. Körner; J.W. Kaldereit; G. Neuhaus y W. Sonnewald. 2008. Lehrbuch der Botanik. Spektrum Akademischer Verlag, Heidelberg, Alemania. pp. 1007-1025.

Casimir, A. 2001. Respuesta del crecimiento y productividad de rábano (Raphanus sativus, L.), cilantro (Coriandrum sativum L.) y habichuela (Phaseolus vulgaris L.) a fertilizante mineral y estiércoles de vaca y oveja en Nigua, República Dominicana. Tesis de maestría. Facultad de Ciencias Agropecuarias y de Recursos Naturales en Diversificación Agrícola. Universidad Pedro Henríquez Ureña, Santo Domingo, República Dominicana.

Cebula, S. 1995. Optimization of plant and shoot spacing in greenhouse production of sweet pepper. Acta Hort. 412, 321-329.

Clavijo, J. 1989. Análisis de crecimiento en malezas. Revista Comalfi 16, 52-63.

Cruz, N.; J. Ortiz; F. Sanchez y M. Mendoza. 2005. Biomasa e índices fisiológicos en chile morrón cultivado en altas densidades. Rev. Fitotec. Mex. 28(3), 287-293.

Gardner, F.; R. Pearce y R. Mitchell. 1985. Physiology of crop plants. Iowa State University Press, Ames, IA.
Gómez, C.; C. Buitrago; M. Cante y C. Huertas. 1999. Ecofisiología de papa Solanum tuberosum utilizada para consumo fresco y para la industria. Revista Comalfi 26(1-3), 42-55.

Hunt, R. 1990. Basic growth análisis. Academic Press, London.

Jolliffe, P.A. y M.M. Gaye. 1995. Dynamic of growth and yield components of bell peppers (Capsicum annum L) to row covers and population density. Scientia Hort. 62, 153-164.

Kerby, A.; K. Cassman y M. Keeley. 1990. Genotypes and plant densities for narrow-row cotton systems. II. Leaf area and dry matter partitioning. Crop Science 30, 649-653.

Kvet, J.; J. Ondok; J. Necas y P. Jarvis. 1971. Methods of growth análisis. Plant photosynthetic production. Manual of methods. W. Junk Publishers, The Hague, The Netherlands.

Lambers, H.; F.S. Chapin III y T.L. Pons. 1998. Plant physiological ecology. Springer, New York, NY.

Lambers, H. y H. Poorter. 1992. Inherent variation in growth rate between higher plants: A search for physiological causes and ecological consequences. Adv. Ecol. Res. 23, 187-261.

Marín, D. 1986. Rendimiento en granos en Canavalia ensiformis (L.) DC. bajo diferentes arreglos espaciales, épocas y densidades de siembra. Rev. Fac. Agron. 14, 205-219.

Marín, D. 1989. Análisis de crecimiento en Canavalia ensiformis (L.) DC. bajo condiciones de campo. Rev. Fac. Agron. 15, 1-16.

Milthorpe, F. y J. Moorby. 1982. Introducción a la fisiología de los cultivos. Hemisferio Sur, Buenos Aires.

Montero, S.M.; B.K. Singh y R. Taylor. 2006. Evaluación de seis estructuras de producción hidropónica diversificada en el trópico húmedo de Costa Rica. Tierra Tropical 2(1), 27-37.

Olalde, V.; J. Escalante; P. Sánchez; L. Tijerina; A. Mastache y E. Carreño. 2000. Crecimiento y distribución de biomasa en girasol en función del nitrógeno y densidad de población en clima cálido. Terra 18, 313-323.

Öpik, H. y S. Rolfe. 2005. The physiology of flowering plants. Cambridge University Press, Cambridge, UK. 
Páez, A.; V. Paz y L. López, J. 2000. Crecimiento y respuestas fisiológicas de plantas de tomate cv. Río Grande en la época mayo-julio. Efecto de sombreado. Rev. Fac. Agron. (LUZ) 17, 173-184.

Perez, M.;L. Carewy N. Battey. 2005. Efectodela densidad de siembra sobre el crecimiento vegetativo y reproductivo de la fresa cv Elsanta. Bioagro 17(1), 11-15.

Poorter, H. 1989. Growth analysis: towards a synthesis of the classical and the functional approach. Physiol. Plant. 75, 237-244.

Poorter, H. y E. Garnier. 1996. Plant growth analysis: an evaluation of experimental design and computational methods. J. Exp. Bot. 47(302), 1343-1351.

Radford, P. 1967. Growth analysis formulae, their use and abuse. Crop Sci. 7(3), 171-175.

Ramírez, R. y M. Pérez, M. 2006. Evaluación del potencial de los sólidos procedentes del tratamiento de aguas residuales para uso agrícola y su efecto sobre el cultivo de rábano rojo (Raphanus sativus, L.). Rev. Fac. Nal. Agr. Medellín 59(2), 3543-3556.

Rincón, A.; G. Ligarreto y D. Sanjuanelo. 2007. Crecimiento del maíz y los pastos (Brachiaria sp.) establecidos en monocultivo y asociados en suelos ácidos del piedemonte llanero colombiano. Agron. Colomb. 25(2), 264-272.

Shibles, R. 1987. Crop physiology. Iowa State University, Iowa, IA.

Taiz, L. y E. Zeiger. 2006. Plant physiology. Sinauer Associates Inc., Sunderland, MA.

Viloria, A.; L. Arteaga y H. Rodríguez. 1998. Efecto de la distancia de siembra en las estructuras de la planta de pimentón. Agronomía Tropical 48(4), 413-423.

Villar, R.; J. Ruiz; J. Quero; H. Poorter; F. Valladares y T. Marañón. 2004. Tasas de crecimiento en especies leñosas: aspectos funcionales e implicaciones ecológicas. pp. 191-227. En: Ecología del bosque mediterráneo en un mundo cambiante. Ministerio de Medio Ambiente; EGRAF, Madrid. 